\title{
Historia natural del cierre percutáneo de foramen oval permeable
}

\author{
Gonzalo Martínez ${ }^{1}$, Marcela Adasme ${ }^{1}$, Juan Francisco Bulnes ${ }^{1}$, Felipe Castañía ${ }^{1}$, Francisco Pacheco ${ }^{1}$, René Hameau ${ }^{1}$, Jorge Quitral ${ }^{1}$, \\ Alberto Fuensalida ${ }^{1}$, Pablo Sepúlveda ${ }^{1}$, Patricio Mellado ${ }^{2}$, Alejandro Martínez ${ }^{1}$. \\ 1 División de Enfermedades Cardiovasculares. \\ 2 Departamento de Neurología. \\ Pontificia Universidad Católica de Chile.
}

Los autores declaran no tener conflictos de interés en relación con este manuscrito.

Financiamiento: ninguno

Introducción: El cierre percutáneo del foramen oval permeable (FOP) se ha posicionado como el tratamiento de elección para la prevención secundaria de pacientes con infartos encefálicos (IE) criptogénicos asociados a FOP.

Objetivo: Revisar los cierres de FOP realizados en nuestra institución, evaluando las características clínicas y del procedimiento, los resultados a mediano plazo luego del procedimiento y la tendencia en el número de intervenciones durante el período estudiado.

Métodos: Se incluyeron 101 pacientes consecutivos en que se realizó cierre de FOP, con una mediana de seguimiento de 4,6 años. Se analizaron las características basales de los pacientes, la indicación del cierre de FOP, el éxito del procedimiento y la presencia de shunt residual en ecocardiografía al año. Se realizó una encuesta telefónica estructurada a todos los pacientes, en la cual se preguntó por nuevo IE o crisis isquémica transitoria (CIT), otros eventos cardiovasculares y la presencia de sangrados. El seguimiento fue completado en el $95 \%$. Se calculó el puntaje RoPE ("Risk of Paradoxical Embolism") el cual provee una estimación de la posibilidad de que ese IE se haya debido al FOP y del riesgo de repetir un nuevo IE en caso de no cerrar el
FOP para cada paciente.

Resultados: La edad promedio fue de 49,1 13,7 años, con 53\% mujeres. Sólo en 3 pacientes se diagnosticó una trombofilia. En 96 pacientes la indicación fue para prevención de embolía paradojal e IE (74\% IE, $17 \%$ CIT y $4 \%$ embolía periférica), mientras que en $5 \%$ por síndrome de ortodeoxia/platipnea. El cierre de FOP fue exitoso en todos los pacientes. Shunt residual en ecocardiograma al año se observó en 5\% - ninguno de estos pacientes presentó un nuevo evento encefálico durante el seguimiento. Se registraron 2 nuevos IE (4 IE por 1000 pacientes/año) y 1 nueva CIT (2 CIT por 1000 pacientes/año) en el seguimiento, con un promedio de presentación de 3,6 años post procedimiento. Esta tasa de eventos fue significativamente menor a lo predicho por el puntaje RoPE en nuestra cohorte. Se observó un marcado aumento en el número de procedimientos desde el año 2017 en adelante. Conclusiones: En nuestra cohorte, el cierre de FOP fue un procedimiento exitoso y seguro. Se asoció a una baja tasa de nuevos eventos cerebrales, marcadamente menor a lo estimado por el puntaje de riesgo actualmente disponible (RoPE).

Palabras Clave: Cierre percutáneo, Foramen oval permeable.

\section{Correspondencia:}

Dr. Gonzalo Martínez Rodríguez

gmartinezr@med.puc.cl 


\section{Natural history of patent foramen ovale's closure}

Introduction: The percutaneous closure of a patent foramen ovale (PFO) has been established as the preferred treatment for those with an ischemic stroke (IS) and associated PFO.

Aims: To review the PFO closure experience at our institution, characterizing the patients and procedures, mid-term results and the trend in the number of interventions during the study period.

Methods: One hundred and one consecutive patients undergoing PFO closure were included, with a median follow-up of 4.6 years. Baseline demographics, PFO closure indications, procedural success rates and residual shunt at 1 -year were recorded. A telephonic survey was performed to complete follow-up, asking for new IS or transient ischemic attacks (TIA), other cardiovascular events and bleeding. Follow-up was completed by $95 \%$. The RoPE score was calculated for each patient, providing an estimate of the chance a given IS being due to a PFO and the risk of a new event when the defect is not closed.

Results: Mean age was 49.1 \pm 13.7 years and 53\% were females. Whereas the indication for PFO closure was paradoxical embolism in 96 patients (74\% IS, $17 \%$ TIA and $4 \%$ peripheral embolism), in 5 it was for platypnea-orthodeoxia syndrome. All patients had a successful PFO closure procedure. Residual shunt at 1 year was found in 5\% - yet, none of these patients experienced a new stroke during the study period. During follow-up there were 2 new IS (4 IS per 1,000 patients/year) and 1 new TIA (2 TIA per 1,000 patients/year), with a mean incidence time of 3.6 years after the procedure. This rate of new events was significantly lower than the one predicted by the RoPE score. From 2017 onwards, there was a marked increase in the number of procedures performed at our institution.

Conclusion: In this cohort, PFO closure was a successful and safe procedure. It was associated to a low rate of new cerebral events during mid-term follow-up, markedly lower than the RoPE predicted rate.

Keywords: Percutaneous closure, Patent foramen ovale. 


\section{Introducción:}

El cierre percutáneo de foramen oval permeable (FOP) se ha establecido como el nuevo estándar de tratamiento en aquellos pacientes que sufren un infarto encefálico (IE) y se identifica un FOP en su estudio, en ausencia de otra causa que explique el evento ${ }^{1}$. Cuatro ensayos clínicos aleatorizados ${ }^{2-5}$ y sus correspondientes meta-análisis 6,7 han mostrado la superioridad de esta intervención por sobre el tratamiento médico. Sin embargo, durante el desarrollo de esta técnica en las últimas 2 décadas la indicación de cierre percutáneo ha pasado por una serie de etapas, desde ser un procedimiento experimental, luego tener resultados negativos en experiencias preliminares, hasta los resultados a favor de la técnica en los ensayos clínicos más recientes. Esto ha sido acompañado de un refinamiento en la técnica utilizada para el cierre, una evolución en los dispositivos utilizados y una mejor selección de los pacientes que más se podrían beneficiar del procedimiento, considerando las características clínicas y anatómicas que confieren un mayor riesgo $^{8}$. Esta evolución experimentada en el cierre percutáneo de FOP ha permitido una progresiva aceptación por parte de la comunidad médica, especialmente neurólogos y cardiólogos, respecto al beneficio conferido por la intervención en pacientes bien seleccionados.

A nivel nacional existe escasa evidencia sobre los resultados obtenidos a lo largo del tiempo con el cierre percutáneo de FOP. En particular, en nuestro conocimiento no existen experiencias publicadas respecto a la evolución de esta técnica con el paso del tiempo, así como tampoco de los resultados obtenidos en términos de prevención secundaria del IE. Por lo tanto, el objetivo de este estudio es revisar los cierres de FOP realizados en nuestra institución, evaluando las características clínicas y del procedimiento y los resultados a mediano plazo luego del procedimiento. Adicionalmente, la información presentada permitirá evaluar la tendencia en la utilización de esta técnica, en relación con la evidencia publicada en la literatura.

\section{Métodos:}

Se incluyeron todos los pacientes en quienes se realizó cierre percutáneo de FOP en nuestra institución hasta diciembre de 2019. Se analizaron las características basales de los pacientes, la indicación del cierre de FOP, el éxito del procedimiento, la presencia de shunt residual en ecocardiografía al año y los eventos clínicos alejados. Se calculó el puntaje $\operatorname{RoPE}^{9}$ para cada paciente, que provee una estimación de la posibilidad de que ese
IE se haya debido al FOP y del riesgo de repetir un nuevo IE en caso de no cerrar el FOP.

\section{Indicación de la intervención y procedimiento:}

El motivo y la indicación de cierre de FOP fue establecida por el equipo tratante, generalmente constituido por un cardiólogo y un neurólogo. Los estudios adicionales de los pacientes con IE fueron también dejados a la decisión del equipo tratante, aunque de acuerdo al protocolo local incluyó imágenes encefálicas, de arterias cervicales y ecocardiograma en prácticamente todos los pacientes. El estudio de trombofilia fue indicado de acuerdo al perfil de riesgo del paciente, realizándose en un $35 \%$ de los casos.

El diagnóstico de FOP se estableció ante la presencia de un shunt de derecha-izquierda a nivel auricular, mediante el uso de solución salina agitada (prueba de burbujas) durante un ecocardiograma transesofágico ${ }^{10}$. La sospecha diagnóstica de esta entidad pudo haberse generado a través de un ecocardiograma de superficie con prueba de burbujas o un Doppler transcraneano, pero en todos los casos se confirmó con el ecocardiograma transesofágico. El cierre percutáneo fue realizado con alguno de los dispositivos comercialmente disponibles, utilizando un acceso venoso femoral y apoyo radioscópico. Adicionalmente, el implante fue realizado con apoyo ecocardiográfico, el cual fue realizado mediante ecocardiograma intracardiaco (ICE) en la mayoría de los casos, sólo bajo anestesia local y sedación superficial. En caso contrario, se utilizó ecocardiograma transesofágico, bajo anestesia general o sedación profunda. La descripción detallada de la técnica de cierre percutáneo de FOP ha sido publicada anteriormente ${ }^{8}$.

De acuerdo a nuestro protocolo local, luego de la intervención los pacientes fueron hospitalizados en una pieza de cuidados básicos. Al día siguiente se dieron de alta, posterior a la realización de un ecocardiograma de superficie para evaluar la correcta posición del dispositivo y la presencia de shunt residual, además de una radiografía de tórax y un electrocardiograma.

\section{Seguimiento:}

Una vez dados de alta, el seguimiento consistió en la evaluación clínica ambulatoria, al menos 2 veces durante el primer año, y la realización de al menos un ecocardiograma de superficie durante este período. Se revisó la ficha clínica de los pacientes en relación al control clínico, sus exámenes y los ecocardiogramas realizados. Se realizó una encuesta telefónica estructurada a 
todos los pacientes, en la cual se preguntó por nuevo IE o Crisis Isquémica Transitoria (CIT), otros eventos cardiovasculares y la presencia de sangrados (de acuerdo con los criterios $\mathrm{BARC}^{11}$ ). El tiempo de seguimiento fue considerado entre la fecha de la intervención y la fecha de la realización de la encuesta telefónica. En caso de fallecimiento, se consideró la fecha de muerte como fin del seguimiento.

\section{Análisis estadístico:}

Las variables categóricas son expresadas como n o \% y las variables continuas como promedio (desviación estándar [DE]). Para el análisis de sobrevida libre de nuevo IE/CIT se incluyeron sólo aquellos pacientes en que la indicación del cierre de FOP fue como prevención secundaria de embolía paradojal, esto es, se excluyeron 5 pacientes en quienes la indicación del cierre fue por ortodeoxia/platipnea. Se utilizó la curva de Kaplan-Meier para análisis de sobrevida libre de eventos. Para el análisis estadístico se utilizó el software SPSS versión 21 (SPSS Inc., Chicago, IL).

\section{Resultados:}

\section{Características clínicas y del procedimiento.}

Entre diciembre de 2005 y diciembre de 2019 se realizaron 101 cierres percutáneos de FOP en nuestra institución (Tabla 1). La edad promedio fue de 49,1 $\pm 13,7$ años, con 53\% mujeres. El 26\% eran hipertensos, $6 \%$ diabéticos y $15 \%$ fumadores. Sólo en 3 pacientes se diagnosticó una trombofilia. En 96 casos, la indicación fue prevención secundaria de embolía paradojal (74\% IE, $17 \%$ CIT y $4 \%$ embolía periférica) y en 5 pacientes por síndrome de ortodeoxia/platipnea.

En aquellos pacientes en los cuales la indicación fue como prevención de embolía paradojal $(n=96)$, el puntaje RoPE promedio fue de 6,7 $(1,6)$, lo cual se traduce en un riesgo estimado de nuevo IE a 2 años de 6,8\%.

$\mathrm{El}$ cierre de FOP fue exitoso en todos los pacientes. Se utilizó un dispositivo de $25 \mathrm{~mm}$ en el $85 \%$ de los casos, utilizando un dispositivo de $30 \mathrm{~mm}$ en los restantes. En cuanto a complicaciones relacionadas con el procedimiento, se registró un pseudoaneurisma de arteria femoral, el cual fue resuelto con compresión guiada por ecografía y prolongó la hospitalización en 24 horas. Dos pacientes presentaron fibrilación auricular paroxística posterior al procedimiento, que cedieron espontáneamente.

Al momento del alta, se indicó Aspirina en $85 \%$ de los pacientes, Clopidogrel en $63 \%$ (60\% con terapia antiagregante dual), estatinas en $47 \%$, anti-hipertensivos en

\begin{tabular}{|c|c|}
\hline \multicolumn{2}{|c|}{ Tabla 1. Características de los pacientes y del procedimient } \\
\hline Edad & $49,1 \pm 13,7$ \\
\hline Mujeres & $53 \%$ \\
\hline Hipertensión arterial & $26 \%$ \\
\hline Diabetes mellitus & $6 \%$ \\
\hline Dislipidemia & $18 \%$ \\
\hline Tabaquismo activo & $15 \%$ \\
\hline Trombofilia & $3 \%$ \\
\hline \multicolumn{2}{|l|}{ Indicación del cierre } \\
\hline IE & $74 \%$ \\
\hline TIA & $17 \%$ \\
\hline Embolía periférica & $4 \%$ \\
\hline Síndrome ortodeoxia/platipnea & $5 \%$ \\
\hline Puntaje RoPE & $6,7(1,6)$ \\
\hline \multicolumn{2}{|l|}{ Tamaño del dispositivo } \\
\hline \#25 & $85 \%$ \\
\hline$\# 30$ & $15 \%$ \\
\hline Shunt residual al año & $5 \%$ \\
\hline \multicolumn{2}{|l|}{ Terapia al alta } \\
\hline Aspirina & $85 \%$ \\
\hline Clopidogrel & $63 \%$ \\
\hline Terapia antiagregante dual & $60 \%$ \\
\hline Anti-hipertensivos & $30 \%$ \\
\hline Estatinas & $47 \%$ \\
\hline Anticoagulación oral & $16 \%$ \\
\hline
\end{tabular}

$30 \%$ y tratamiento anticoagulante oral en $16 \%$.

\section{Seguimiento:}

El seguimiento fue completado, con la encuesta telefónica, en el $95 \%$ de los casos. La mediana de seguimiento fue de 4,6 años. Cuatro pacientes presentaron un episodio clínico de trombosis venosa profunda en el seguimiento, sin evidencias de embolía paradojal. Ocurrieron 7 episodios de sangrado que requirieron atención médica - 1 BARC 3c, 1 BARC 3b, 2 BARC 3a y 3 BARC 2 - con un caso de hemorragia encefálica (paciente en tratamiento con aspirina). Se observó shunt residual en el ecocardiograma de superficie al año en $5 \%$ de los pacientes, sin embargo, ninguno de estos pacientes presentó un nuevo evento cerebral. No se re-in- 
tervino a ningún paciente. Se registraron 2 muertes en el seguimiento, ambas de causa no cardiovascular, y en quienes el cierre de FOP fue indicado por síndrome de platipnea/ortodeoxia.

Se registraron 2 nuevos IE (4 IE por 1000 pacientes/ año) y 1 nueva CIT (2 CIT por 1000 pacientes/año) en el seguimiento, con un promedio de presentación de 3,6 años - ninguno ocurrió antes de los 2 años post intervención. De acuerdo al puntaje RoPE, el riesgo estimado de nuevo IE a 2 años fue de $6,8 \%$. Si consideramos el peor escenario, esto es asumiendo que el riesgo de nuevo IE estimado por RoPE es de $6,8 \%$ durante todo el seguimiento de nuestro estudio (es decir 4,6 años en vez de 2 años), el cierre de FOP resultó en una disminución teórica de $58 \%$ del riesgo esperado de IE/CIT recurrente en nuestra cohorte (Figura 1).

Tendencia temporal en el cierre percutáneo de FOP. En un período de 15 años se realizaron 101 cierres percutáneos de FOP. Sin embargo, la distribución temporal fue muy heterogénea, con una tendencia al aumento de casos anuales durante los últimos años de este período, ya que el $45 \%$ de los casos se concentraron en los últimos 3 años. La figura 2 muestra el número de casos anuales realizados, en el contexto de la evidencia acumulada respecto a esta técnica.

\section{Discusión:}

El presente trabajo confirma, en una experiencia chilena, los buenos resultados inmediatos y a mediano plazo del cierre percutáneo de FOP. Adicionalmente, mediante el análisis de la tendencia temporal de los procedimientos y su relación con la literatura publicada, permite vislumbrar como esta técnica se ha establecido como un estándar en aquellos pacientes que sufren un IE/CIT sin causa identificable y asociado a la presencia de un FOP. De este modo, es esperable que experimentemos un aumento considerable en la indicación de este procedimiento, como se puede inferir del marcado incremento en nuestra institución durante los últimos 3 años.

Tres grandes ensayos clínicos recientes han inclinado la balanza a favor del cierre percutáneo de FOP como

Figura 1. Aparición de nuevo IE/CIT luego de cierre percutáneo de FOP.

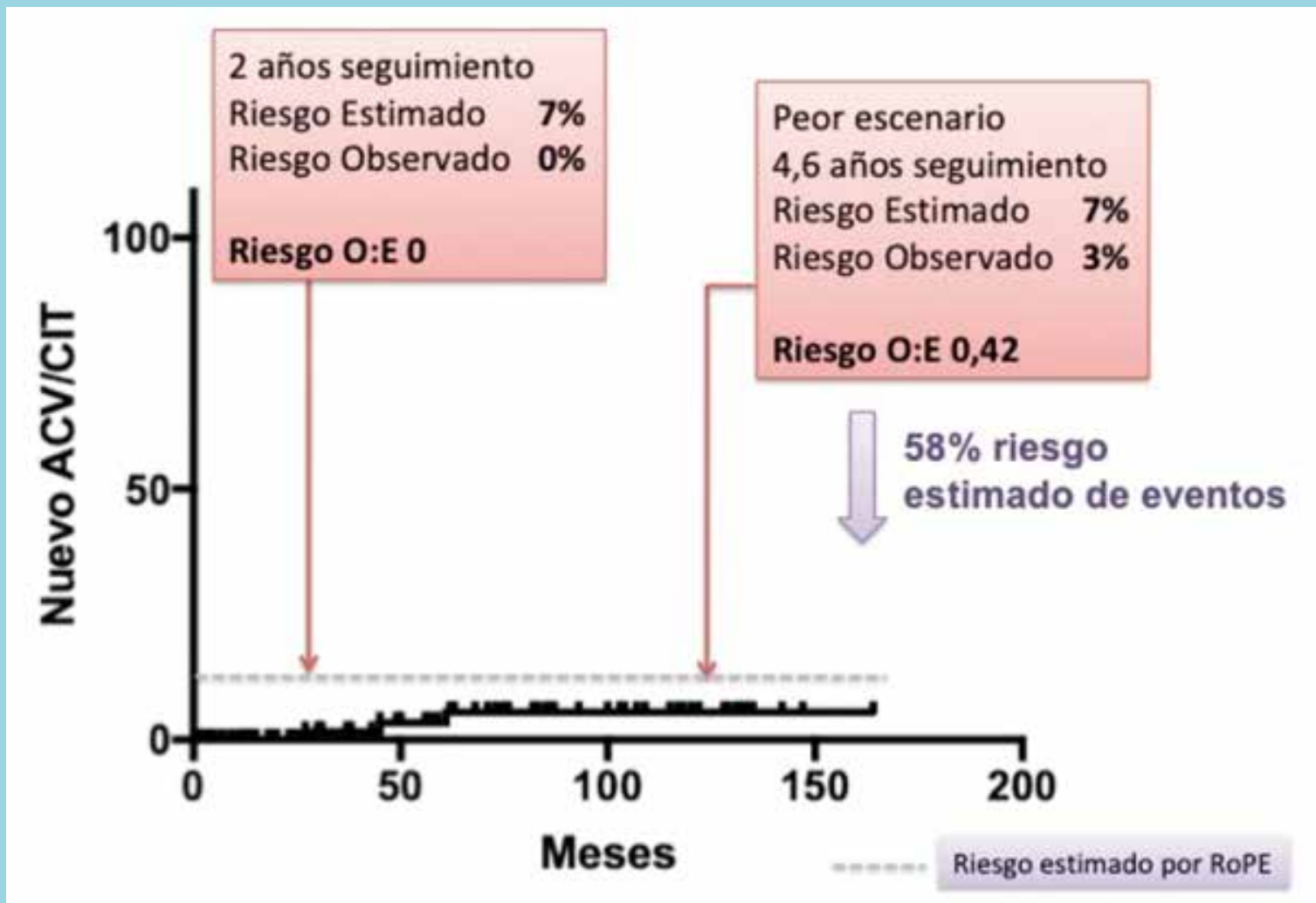




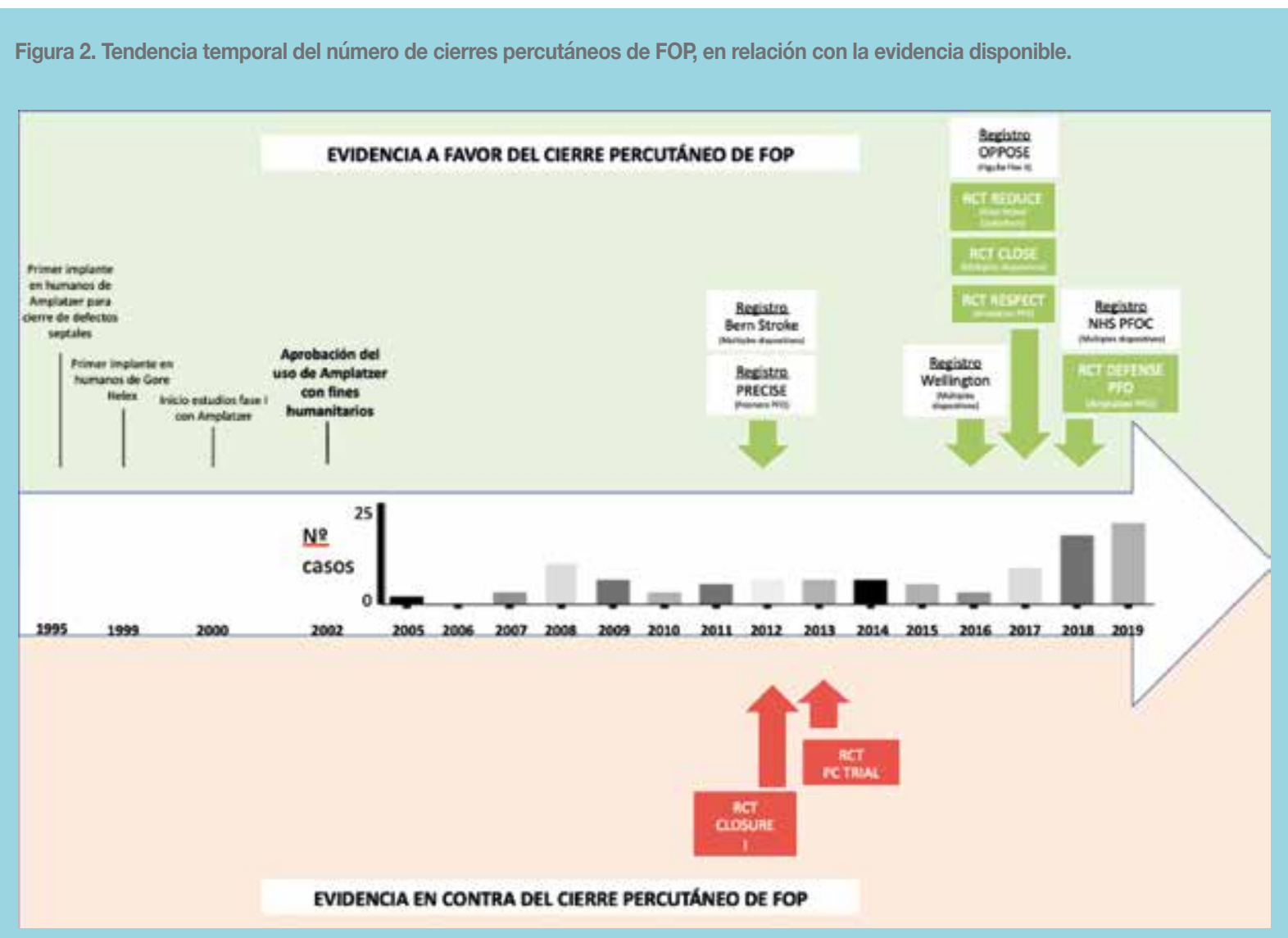

terapia de prevención secundaria en aquellos pacientes con IE criptogénico y la presencia de un FOP. RES$\mathrm{PECT}^{4}$ fue un estudio financiado por St Jude Medical realizado en Estados Unidos y Canadá. Se incluyeron 980 pacientes, aleatorizados a cierre de FOP (utilizando un Amplatzer PFO Occluder) o tratamiento médico (antiplaquetarios o anticoagulantes). Luego de un seguimiento de 5,2 años, el cierre de FOP se asoció a una reducción de un $42 \%$ en la tasa de nuevos IE, comparado con el tratamiento médico. REDUCE ${ }^{2}$ fue un estudio financiado por el desarrollador de un dispositivo (W.L. Gore And Associates), multinacional, que incluyó 664 pacientes con ACV y FOP y los aleatorizó 1:1 a cierre percutáneo con los dispositivos Gore (GORE® HELEX ${ }^{\circledR}$ Septal Occluder / GORE® CARDIOFORM Septal Occluder) o antiplaquetarios. Luego de un seguimiento de 3,2 años, la incidencia de nuevos IE clínicos fue de $1,4 \%$ vs. 5,4\% en los grupos de cierre de FOP $\mathrm{y}$ antiagregantes plaquetarios, respectivamente. Finalmente, $\mathrm{CLOSE}^{3}$ fue un estudio financiado por el Ministerio de Salud Francés, el cual incluyó 663 pacientes aleatorizados 1:1:1 a cierre de FOP, antiagregación pla- quetaria o anticoagulantes orales. Se utilizaron 11 dispositivos distintos. En un seguimiento de algo más de 5 años, no se observó ningún caso de nuevo IE en el grupo asignado a cierre de FOP, mientras que este evento ocurrió en 14 pacientes asignados a terapia antiplaquetaria. La terapia anticoagulante protegió efectivamente contra nuevos eventos en comparación con la antiagregación plaquetaria, pero no a los niveles del cierre de FOP. En su conjunto, esta evidencia favorece el cierre de FOP en esta población (prevención secundaria del IE sin otra causa), particularmente cuando el defecto se acompaña de algún marcador ecocardiográfico de mayor riesgo, principalmente, un septum interauricular aneurismático o un tamaño del defecto grande (caracterizado como un shunt derecha-izquierda abundante) ${ }^{5}$.

Estos resultados contradicen los estudios negativos previos, debido principalmente a un cambio en la inclusión de los pacientes, que hace más probable que el FOP esté vinculado al evento neurológico. Así, en los nuevos estudios se incluyeron pacientes menores de 60 años, con IE de tipo embólico (no lacunar), sin causa cardíaca ni enfermedad de los vasos del cuello y con un FOP anató- 
micamente de riesgo ${ }^{12}$.

De forma interesante, en nuestra experiencia encontramos una tendencia temporal de los procedimientos similar a lo reportado internacionalmente ${ }^{13}$, con un aumento de los casos hasta el año 2009, favorecido por el entusiasmo de la comunidad intervencionista en relación con los resultados de los primeros estudios pilotos, seguido de una caída en el número de procedimientos luego de la aparición de los primeros estudios negativos, y nuevo incremento en los últimos 3 años.

La consolidación de esta evidencia y la penetrancia que ha tenido en la comunidad de cardiólogos y neurólogos que evalúan a estos pacientes de forma cotidiana ha hecho incluso plantear nuevas recomendaciones de cómo categorizar a estos enfermos. Es así como actualmente se propone que en casos de IE superficiales, profundos grandes o retinales, sin otra causa evidente, y con un FOP de moderado-alto riesgo, se debiera usar el término IE asociado a FOP, eliminando el término criptogénico que aduce al desconocimiento de la causa ${ }^{14}$.

En aquellos pacientes en quienes se indicó cierre de FOP para prevención de embolía sistémica, encontramos que la tasa de nuevos eventos embólicos (IE/CIT u otro) fue de 6 eventos cada 1000 pacientes/año. Esto es marcadamente menor que lo predicho de acuerdo al puntaje RoPE de los pacientes intervenidos, donde la tasa de eventos esperados sin intervención hubiese sido de 32 eventos cada 1000 pacientes/año. Más aún, ninguno de los pacientes intervenidos tuvo un nuevo evento durante los primeros 2 años, el cual es el tiempo evaluado por este puntaje. Estos resultados son concordantes con registros previos 15,16 .

Del mismo modo, la población intervenida en nuestra serie parece reflejar el perfil de los pacientes intervenidos en la práctica clínica cotidiana. En un registro canadiense de más de 200 pacientes, Wintzer-Wehekind y cols mostraron los resultados a largo plazo (promedio 12 años) de esta técnica en una población muy similar a la nuestra ${ }^{16}$. La edad promedio de esta cohorte fue de 47 años, con $51 \%$ mujeres, un puntaje de RoPE de 6,6 y con IE/CIT previo en $76 / 32 \%$ (comparado con 49 años, 53\% mujeres, RoPE 6,7 e IE/CIT previo en $74 / 17 \%$ en nuestra serie). Los resultados presentados en esta serie canadiense son excelentes, con una muy baja tasa de nuevos eventos en el seguimiento alejado. Adicionalmente, el perfil de los pacientes intervenidos en nuestra serie se asemeja bastante al de los pacientes incluidos en los estudios más recientes que han demostrado el beneficio del cierre percutáneo (RESPECT, REDUCE y CLOSE). Llama la atención, no obstante, el bajo número de pacientes con trombofilia diagnosticada en nuestra cohorte (sólo 3 casos; en el registro canadiense fue de $15 \%$ ). Es posible que esto se deba a que durante gran parte del tiempo en nuestra institución se excluyó a aquellos pacientes con trombofilia del cierre de FOP, dado que requerirían terapia anticoagulante de forma permanente de todas formas. Esta concepción ha ido variando lentamente en base a la nueva evidencia.

Dado el carácter retrospectivo de nuestro registro, los resultados presentan varias limitaciones. Desde luego, la identificación de nuevos eventos isquémicos puede verse afectada en un estudio de esta naturaleza. Sin embargo, el haber realizado una encuesta telefónica a casi todos los pacientes permitió obtener información más fidedigna de la incidencia de nuevos eventos durante el seguimiento. Segundo, la evidencia actual favorece la caracterización del riesgo de los FOP de acuerdo a sus características anatómicas ${ }^{17}$. Lamentablemente, en el comienzo de nuestra experiencia, y por un período largo, estas características no se encontraban del todo claras y estaban pobremente definidas, de modo que no contamos con un registro adecuado del tamaño del shunt y la presencia de aneurisma del septum en el ecocardiograma pre-procedimiento, lo cual nos hubiese permitido caracterizar mejor la población intervenida en nuestra cohorte. Tercero, es posible que exista un sub-registro de complicaciones menores, como fibrilación auricular paroxística en relación con el procedimiento. Esto no parece haber afecto los resultados, sin embargo, dado que en ninguno de los 3 casos nuevos de IE/CIT en el seguimiento se encontró fibrilación auricular como agente etiológico, aún después del estudio pertinente.

En conclusión, en nuestra cohorte el cierre percutáneo de FOP fue un procedimiento exitoso y seguro. Se asoció a una baja tasa de nuevos eventos encefálicos, marcadamente menor a lo estimado por el puntaje de riesgo actualmente disponible (RoPE). La característica de la población intervenida y los resultados obtenidos están en consonancia con los publicados en la literatura y han resultado en un marcado aumento en la realización de esta intervención en los últimos años. 


\section{Referencias}

1. KUIJPERS T, SPENCER FA, SIEMIENIUK RAC, et al. Patent foramen ovale closure, antiplatelet therapy or anticoagulation therapy alone for Management of cryptogenic stroke? A clinical practice guideline. BMJ 2018; 362:k2515.

2. SØNDERGAARD L, KASNER SE, RHODES JF, et al. Patent foramen ovale closure or antiplatelet therapy for cryptogenic stroke. N Engl J Med 2017; 377:1033-42.

3. MAS J-L, DERUMEAUX G, GUILLON B, et al. Patent foramen ovale closure or anticoagulation vs. antiplatelets after stroke. N Engl J Med 2017; 377:1011-21.

4. SAVER JL, CARROLL JD, THALER DE, et al. Long-Term outcomes of patent foramen ovale closure or medical therapy after stroke. N Engl J Med 2017; 377:1022-32.

5. LEE PH, SONG J-K, KIM JS, et al. Cryptogenic stroke and high-risk patent foramen ovale: the DEFENSE-PFO trial. J Am Coll Cardiol 2018; 71:2335-42.

6. VADUGANATHAN M, QAMAR A, GUPTA A, et al. Patent foramen ovale closure for secondary prevention of cryptogenic stroke: updated metanálisis of randomized clinical trials. Am J Med 2018; 131:575-7.

7. AHMAD Y, HOWARD JP, ARNOLD A, et al. Patent foramen ovale closure vs. medical therapy for cryptogenic stroke: a meta-analysis of randomized controlled trials. Eur Heart J 2018; 39:1638-49.

8. HORLICK E, KAVINSKY CJ, AMIN Z, et al. SCAI expert consensus statement on operator and institutional requirements for PFO closure for secondary prevention of paradoxical embolic stroke. Catheter Cardiovasc Interv. 2019; 93:859874 .

9. KENT DM. RUTHAZER R, WEIMAR C. An index to identify stroke-related vs incidental patent foramen ovale in cryptogenic stroke. Neurology. 2013; 81(7):619-25.
10. CABANES L, COSTE J, DERUMEAUX G, et al. Interobserver and intraobserver variability in detection of patent foramen ovale and atrial septal aneurysm with transesophageal echocardiography. J Am Soc Echocardiogr 2002; 15:441-6.

11. MEHRAN R, RAO SV, BHATT DL, et al. Standardized bleeding definitions for cardiovascular clinical trials: a consensus report from the Bleeding Academic Research Consortium. Circulation 2011; 123:2736-47.

12. WIKTOR DM, CARROLL, JD. The Case for Selective Patent Foramen Ovale Closure After Cryptogenic Stroke. Circ Cardiovasc Interv. 2018; 11:e04152.

13. FAROOQI M, STICKLEY J, DHILLON R, et al. Trends in surgical and catheter interventions for isolated congenital shunt lesions in the UK and Ireland. Heart 2019; 0:1-6

14. ELGENDY AY, SAVER JL, AMIN Z, et al. Proposal for updated nomenclature and classification of potential causative mechanism in patent foramen ovale-associated stroke. JAMA Neurology. 2020; Epub ahead of print.

15. RIGATELLI G, PEDON L, ZECCHEL R, et al. Long term outcomes and complications of intracardiac echocardiography-assisted patent foramen ovale closure in 1,000 consecutive patients. J Intervent Cardiol 2016; 29:530-8.

16. WINTZER-WEHEKIND J, ALPERI A, HOUDE C, et al. Long-Term Follow-Up After Closure of Patent Foramen Ovale in Patients With Cryptogenic Embolism. J Am Coll Cardiol 2019; 73:278-87.

17. SILVESTRY FE, COHEN MS, ARMSBY LB, et al. American Society of Echocardiography; Society for Cardiac Angiography and Interventions. Guidelines for the Echocardiographic Assessment of Atrial Septal Defect and Patent Foramen Ovale: from the American Society of Echocardiography and Society for Cardiac Angiography and Interventions. J Am Soc Echocardiogr. 2015; 28:910-958. 